\title{
Berechnung optimaler Ausgangsrückkopplungen zur Lösung von Multimodellproblemen
}

\author{
Herrn Professor Jürgen Ackermann zur Vollendung des 60. Lebensjahres gewidmet
}

\author{
Andras Varga und Georg Grübel, Oberpfaffenhofen
}

Dr.-Ing. Andras Varga ist wissenschaftlicher Mitarbeiter am DLR - Institut für Robotik und Systemdynamik, Oberpfaffenhofen, Abteilung Entwurfsorientierte Regelungstechnik (Prof. G. Grübel). Hauptarbeitsfelder: Numerische Verfahren der Systemtheorie, Ordnungsreduktion, Deskriptorsysteme, Periodische Systeme, Rechnergestützte Analyse- und Entwurfsverfahren.

Addresse: DLR - Oberpfaffenhofen

Deutsche Forschungsanstalt für Luft- und Raumfahrt

Institut für Robotik und Systemdynamik

Postfach 1116, D-82230 Wessling.

E-mail: Andreas.Varga@dlr.de

Prof. Dr.-Ing. Georg Grübel ist Leiter der Abteilung Entwurfsorientierte Regelungstechnik im DLR - Institut für Robotik und Systemdynamik, Oberpfaffenhofen, und apl. Professor für Regelungstechnik im Institut für Automatisierungstechnik der Fakultät für Maschinenbau der Ruhr-Universität Bochum.

Addresse: siehe oben

E-mail: Georg.Gruebel@dlr.de

Im Aufgabenbereich "robuste Regelung" stellt sich das Problem (dynamische) Ausgangsrückkopplungen zu bestimmen, die mehrere lineare Systeme simultan stabilisieren. Eine Methode dafür, nämlich die Berechnung linearer quadratisch-optimaler Ausgangsrückkopplungen, wird hier behandelt. Effiziente numerische Algorithmen in verfügbarer Software sowohl zur Parameteroptimierung als auch für die dabei notwendigen Auswertungen von Gütefunktional und Gradient mittels Liapunovgleichungen, machen diese Methode für "große" Multimodellprobleme anwendbar. Dies gilt insbesondere auch für die Berechnung diskret-periodischer optimaler Ausgangsrückkopplungen, wo die Problemgröße mit der Periodenzahl wächst. 


\section{Computation of optimal output feedback control for solving multi-model pro- blems}

In the domain of "robust control" the problem arises how to compute (dynamic) outputfeedback laws, which simultaneously stabilize multi models. One among possible solution methods - namely linear quadratic-optimal output feedback is dealt with. Efficient numerical algorithms in available software for both parameter optimization and the evaluation of the performance functional and its gradient by solving Liapunov equations make this method applicable for "large" multi-model problems. This also holds for the computation of discrete optimal periodic output feedback, where the problem dimension increases with the period of the system.

\section{Einführende Übersicht}

Die Kalmansche Theorie der linearen quadratisch-optimalen Regelung führt über den bekannten Riccatiformalismus [10] auf ein Regelgesetz mit vollbesetzter Zustandsrückkopplung. Eine dazu verallgemeinerte Aufgabenstellung ist die lineare quadratisch-optimale Regelung mit strukturbeschränkter Rückkopplung [8],[14]. Eine Spezialisierung der strukturbeschränkten Rückkopplung auf eine Ausgangsgrößenrückkopplung erfolgte in [16]. Über die Möglichkeit, die Struktur des Regelgesetzes explizit festlegen zu können, sind neben einer direkten Ausgangsrückkopplung auch verschiedenartige Aufgabenstellungen von Regelgesetzen mit Dynamik behandelbar [7].

Ein lineares quadratisch-optimales Regelgesetz mit beschränkter Struktur kann in der Regel nicht in expliziter Form angegeben werden, sondern es müssen numerische Iterationsverfahren zur Bestimmung der Rückführmatrix angewendet werden [18]. Allerdings können die dazu notwendigen Gradienten analytisch bestimmt werden [8]. Für die effiziente Auswertung des Gütefunktionals und des Gradienten sind leistungsfähige Algorithmen $[2,12]$ zur Lösung von Liapunovgleichungen erforderlich. Eine gute Iterationskonvergenz kann durch Einsatz geeigneter Parameteroptimierungsprogramme [17] mit Schrittweitensteuerung [20] sichergestellt werden.

Eine lineare quadratisch-optimale (dynamische) Ausgangsrückkopplung ist einerseits deshalb attraktiv, weil sie sehr flexibel an verschiedenartige Aufgabenstellungen anpaßbar ist und weil sie andererseits vergleichsweise effizient numerisch berechenbar ist.

Die Möglichkeit die Struktur der Rückkopplungsmatrix explizit festlegen zu können, ist insbesondere eine Voraussetzung dafür, parameterunempfindliche [4] und robuste Regelungen durch Lösung eines sogenannten "Multimodellproblems" zu entwickeln. Das ist das Thema dieses Aufsatzes. Dabei wird die Aufgabenstellung des zeitinvarianten Multimodellproblems auch auf ein Multimodellproblem mit diskret-periodischer Ausgangsrückkopplung [26] erweitert. Das Multimodellproblem, siehe auch Ackermann [1], kann daraus entstehen, daß ein nichtlineares System durch Linearisierungen in mehreren Arbeitspunkten charakterisiert wird (arbeitspunktrobuste Regelung) oder, daß die Parameterunsicherheit eines Systems durch mehrere diskrete Werte in einem Intervall erfaßt werden soll (robuste Regelung mit strukturierter Parameterunsicherheit).

Die Möglichkeit eine lineare quadratisch-optimale Ausgangsrückkopplung effizient zu berechnen, macht diese Methode auch für "große" Multimodellprobleme anwendbar, d.h. 
für Aufgaben, wo eine stabilisierende Lösung simultan für "viele" lineare Modelle zu ermitteln ist, die aus der Linearisierung eines nichtlinearen Systems in entsprechend vielen Arbeitspunkten resultieren. Ein Multimodellproblem kann auch dadurch "groß" werden, daß ein Multimodellsystem durch eine periodische Ausgangsrückkopplung simultan stabilisiert werden soll und dafür die Periodenzahl hinreichend groß gewählt werden muß.

Eine "große" Multimodellaufgabe stellt sich bei der Untersuchung, wie die Bodenresonanzeffekte eines Hubschraubers aktiv gedämpft [23], [11] werden können. Diese Aufgabe war Anlaß, sich mit "großen" Multimodellproblemen zu befassen und dient hier als Anwendungsbeispiel.

\section{Zur Berechnung linearer quadratisch-optimaler Ausgangsrückkopplungen}

Das Modell einer linearen, zeitinvarianten Regelstrecke sei durch

$$
\begin{aligned}
\lambda x(t) & =A x(t)+B u(t) \\
y(t) & =C x(t)+D u(t)
\end{aligned}
$$

beschrieben, wobei $x(t) \in \mathbb{R}^{n}$ der Zustandvektor, $u(t) \in \mathbb{R}^{m}$ der Eingangsvektor und $y(t) \in \mathbb{R}^{p}$ der Ausgangsvektor sind. Der Operator $\lambda$ ist entweder der Differentialoperator $\lambda x(t)=\dot{x}(t)$ im Fall eines kontinuierlichen Systems oder der Vorwärtsverschiebungsoperator $\lambda x(t)=x(t+1)$ im Fall eines diskreten Systems.

Die Aufgabe ist es, eine optimale Ausgangsrückkopplung

$$
u^{*}(t)=F y(t)
$$

$\mathrm{zu}$ bestimmen, die das folgende quadratische Gütefunktional ${ }^{1}$ minimiert, wobei $Q \geq 0$ und $R>0$ symmetrische Wichtungsmatrizen sind:

- für ein zeitkontinuierliches System

$$
J=E\left\{\int_{0}^{\infty}\left[x(t)^{T} Q x(t)+u(t)^{T} R u(t)\right] d t\right\}
$$

- für ein zeitdiskretes System

$$
J=E\left\{\sum_{t=0}^{\infty}\left[x(t)^{T} Q x(t)+u(t)^{T} R u(t)\right]\right\} .
$$

Der Wert des Gütefunktionals $J$ hängt von den Anfangsbedingungen $x(0)$ des Systems ab. Für eine Standardlösung kann man $x(0)$ als Zufallsvektor mit der Kovarianz $G=$ $E\left\{x(0) x^{T}(0)\right\}$ betrachten. Entsprechend ist das Gütefunktional als Erwartungsoperator $E\{\cdot\}$ definiert.

\footnotetext{
${ }^{1}$ Kuhn [15] verwendet einen anderen Typ eines quadratischen Funktionals, bei dem an Stelle einer Wichtung der Stellgrößen die Rückführverstärkungen direkt gewichtet werden.
} 
Für die Berechnung kann $D=0$ vorausgesetzt werden. Die Lösung im allgemeinen Fall $D \neq 0$ ist

$$
F=(I+\widetilde{F} D)^{-1} \widetilde{F}
$$

wobei $\widetilde{F}$ die Rückführmatrix für den speziellen Fall $D=0$ ist. Dies gilt unter der Voraussetzung, daß $I+\widetilde{F} D$ invertierbar ist.

Sei weiter $F$ eine stabilisierende Rückführmatrix des Systems (1). Das heißt, die Eigenwerte $\lambda_{i}(\bar{A})$ des geschlossenen Systems $\bar{A}=A+B F C$ erfüllen die Bedingung $\operatorname{Re} \lambda_{i}(\bar{A})<0$ im Fall eines kontinuierlichen Systems oder $\left|\lambda_{i}(\bar{A})\right|<1$ im Fall eines diskreten Systems. Mit der Notation $\bar{Q}=Q+C^{T} F^{T} R F C$ kann dann der Funktionswert des Gütefunktionals und sein Gradientoperator wie folgt berechnet werden, wobei "tr" (engl. trace) die Spur einer Matrix bezeichnet:

- für ein zeitkontinuierliches System

$$
\begin{aligned}
J(F) & =\operatorname{tr}(P G) \\
\nabla_{F} J(F) & =2\left(B^{T} P+R F C\right) S C^{T}
\end{aligned}
$$

mit den "kontinuierlichen Liapunovgleichungen" für $P$ und $S$

$$
\begin{aligned}
& \bar{A}^{T} P+P \bar{A}+\bar{Q}=0 \\
& \bar{A} S+S \bar{A}^{T}+G=0
\end{aligned}
$$

- für ein zeitdiskretes System

$$
\begin{aligned}
J(F) & =\operatorname{tr}(P G) \\
\nabla_{F} J(F) & =2\left(B^{T} P \bar{A}+R F C\right) S C^{T}
\end{aligned}
$$

mit den "diskreten Liapunovgleichungen" für $P$ und $S$

$$
\begin{aligned}
P & =\bar{A}^{T} P \bar{A}+\bar{Q} \\
S & =\bar{A} S \bar{A}^{T}+G .
\end{aligned}
$$

Mit den expliziten analytischen Ausdrücken für den Funktionswert $J(F)$ und den Gradienten $\nabla_{F} J(F)$ ist die Anwendung eines iterativen Minimierungsverfahrens unmittelbar möglich. Das Minimierungsverfahren muß nur noch durch eine stabilisierende Startlösung $F_{0}$ initialisiert werden. Ist $A$ stabil, dann genügt $F_{0}=0$ zur Initialisierung der Minimierungsprozedur. Ist $A$ instabil, gibt es mehrere Möglichkeiten [18] zur Berechnung einer stabilisierenden Rückführmatrix $F_{0}$. Der einfachste und bequemste Weg ist die Anwendung der Minimierungsprozedur selbst. Im Fall eines kontinuierlichen Systems löst man dazu mit $F_{0}=0$ sukzessiv eine Folge modifizierter Probleme, bei denen $A$ durch $A+\alpha_{j} I$, $j=1,2, \ldots$, ersetzt wird und $\left\{\alpha_{j}\right\}$ eine monoton steigende Folge bildet. Einer der sukzessiv berechneten Werte $F_{j}$, die die Matrix $A+\alpha_{j} I$ stabilisieren, stabilisiert dann auch die Matrix $A$, wenn dafür ein positives $\alpha_{j}$ gilt. Ein entsprechender Ansatz gilt zur Stabilisierung eines diskreten Systems. Diesmal ersetzt man das Paar $(A, B) \operatorname{durch}\left(\alpha_{j} A, \alpha_{j} B\right)$, wobei $\left\{\alpha_{j}\right\}$ eine monoton steigende Folge bildet, die für einen sukzessiv berechneten Wert $F_{j}$, mit $F_{0}=0$, zur Stabilität von $\alpha_{j} A+\alpha_{j} B F_{j-1} C$ (im diskreten Sinn) führt. Eine stabilisierende Rückführung für A ist erreicht, wenn für diesen Schritt $\alpha_{j} \geq 1$ gilt. 
Ein wichtiger Aspekt für die Anwendungsflexibilität der Methode ist das systematische Umgehenkönnen mit Strukturvorgaben für die Rückführmatrix F. Dies ist insbesondere auch eine Voraussetzung für die Behandlung des Multimodellproblems. Strukturvorgabe bedeutet hier die Forderung, daß einzelne Elemente der Matrix F vorgegebene Werte, z.B. null, annehmen. Dies bedeutet für die Minimierungsaufgabe, daß nur die als aktiv gesetzten Komponenten der Rückführmatrix F verändert werden dürfen. Für die Berechnung bedeutet dies, daß bei der Minimierung diejenigen Elemente der Rückführmatrix durch eine Projektion des Gradienten eliminiert werden müssen, für die bereits feste Werte vorgegeben sind. Die Parametersuche wird damit auf eine strukturierte Untermenge der Elemente von $\mathrm{F}$ beschränkt. Der allgemeine mathematische Formalismus zur analytischen Herleitung der entsprechenden Gradienten ist die Methode der sogenannten "Gradientenkettenregeln", wie sie von Höfler in [7] entwickelt wurde. Für die spezielle Struktur einer dezentralen Ausgangsrückkopplung wurden explizite Formeln auch in [24] angegeben.

Der wesentliche numerische Aufwand zur Bestimmung einer optimalen Ausgangsrückkopplung besteht in der Lösung von zwei Liapunovgleichungen für jede Auswertung der Funktion und des zugehörigen Gradienten. Effiziente softwaretechnische Implementierungen für Liapunovlöser, basierend auf den Algorithmen aus [2, 12], sind in den Programmbibliotheken RASP und SLICOT verfügbar [5]. Für die Minimierung verwenden wir Programme aus MINPACK 2 (das Nachfolgepaket von MINPACK 1 [19]). Diese Programme sind Implementierungen des limited memory BFGS Verfahrens aus [17] im Zusammenspiel mit der Schrittweitensteuerungsprozedur aus [20], die bei jedem Schritt eine Verminderung des Funktionswertes garantiert. Diese Eigenschaft einer Schrittweitensteuerung ist notwendig, um eine mögliche Destabilisierung des Systems in einem Zwischenschritt zu vermeiden. Tritt infolge eines zu großen Schritts die Situation einer möglichen Destabilisierung ein, wird einfach der Funktionswert höher als vorher gesetzt und der Schritt wird automatisch aufgegeben. Die softwaretechnische reverse-communication Implementierung in MINPACK 2 erlaubt eine Modularisierung der Berechnungssoftware in eine allgemeine Optimierungssoftware einerseits und die Software zur problemspezifischen Auswertung von Gütefunktional und Gradient andererseits.

\section{Lineare quadratisch-optimale Ausgangsrückkopp- lung für Multimodellprobleme}

Wird ein nichtlineares System in verschiedenen Arbeitspunkten linearisiert, können die resultierenden Systemmodelle zu einem linearen Multimodell zusammengefaßt werden, das aus $N$ Teilmodellen besteht:

$$
\begin{aligned}
\lambda x_{i}(t) & =A_{i} x_{i}(t)+B_{i} u_{i}(t) \\
y_{i}(t) & =C_{i} x_{i}(t)+D_{i} u(t)
\end{aligned}, \quad i=1, \ldots, N
$$

mit $x_{i}(t) \in \mathbb{R}^{n}, u_{i}(t) \in \mathbb{R}^{m}, y_{i}(t) \in \mathbb{R}^{p}$. Des weiteren treffen wir die Annahmen $D_{i}=$ $D=0$ (siehe Abschnitt 2) und es sei $x_{i}(0)$ ein Zufallsvektor mit gegebener Kovarianz $G_{i}=E\left\{x_{i}(0) x_{i}^{T}(0)\right\}$.

Eine alternative Beschreibung des Multimodells (9) ist durch eine Folge von System- 
matrizen

$$
S_{i}=\left[\begin{array}{cc}
A_{i} & B_{i} \\
C_{i} & D_{i}
\end{array}\right], \quad i=1, \ldots, N
$$

charakterisiert. Ein solches Modell gehört zur allgemeineren Klasse sogenannter polytopischer Modelle

$$
\begin{aligned}
\lambda x(t) & =A(t) x(t)+B(t) u(t) \\
y(t) & =C(t) x(t)+D(t) u(t)
\end{aligned}
$$

deren zeitvariable Systemmatrizen $S(t)=\left[\begin{array}{ll}A(t) & B(t) \\ C(t) & D(t)\end{array}\right]$ in einem Polytop von Matrizen

$$
S(t) \in \operatorname{Co}\left\{S_{1}, \ldots, S_{N}\right\}:=\left\{\sum_{i=1}^{N} \alpha_{i} S_{i}: \alpha_{i} \geq 0, \sum_{i=1}^{N} \alpha_{i}=1\right\}
$$

variieren. Die Systeme $S_{1}, \ldots, S_{N}$ werden Trägersysteme (engl. vertex systems) genannt.

Zum Aufgabenbereich stabilitätsrobuster Regelungen gehört es, eine Ausgangsrückkopplung zu bestimmen, die das polytopische System (12) stabilisiert. Eine notwendige Bedingung dafür ist, daß das Multimodellsystem (9) simultan stabilisiert wird, d.h., daß auch alle Ecken des Polytops stabilisiert werden. Die simultane Stabilisierung von Multimodellsystemen spielt deshalb in der Theorie der robusten Regelung eine zentrale Rolle, siehe Ackermann [1].

Die Methode der linearen quadratisch-optimalen Regelung kann dazu dienen eine stabilisierende Multimodellrückkopplung zu berechnen (wenn eine solche existiert), wobei über eine geeignete Wahl der Wichtungmatrizen $Q_{i}$ und $R_{i}$ gleichzeitig auch die Regelgüte beeinflußt werden kann. Die Aufgabe in diesem Fall ist es also, eine optimale Ausgangsrückkopplung der Form (2) zu bestimmen, die simultan alle $N$ Systeme in (9) stabilisiert und ein Gütefunktional $J$ minimiert, das alle $N$ Einzelsysteme im Sinne von (3) bzw. (4) erfaßt:

- für ein zeitkontinuierliches System:

$$
J=E\left\{\int_{0}^{\infty}\left[x(t)^{T} \mathcal{Q} x(t)+u(t)^{T} \mathcal{R} u(t)\right] d t\right\}
$$

- für ein zeitdiskretes System:

$$
J=E\left\{\sum_{t=0}^{\infty}\left[x(t)^{T} \mathcal{Q} x(t)+u(t)^{T} \mathcal{R} u(t)\right]\right\},
$$

mit den blockdiagonalen Matrizen $\mathcal{Q}$ und $\mathcal{R}$ der Form

$$
\mathcal{Q}=\operatorname{diag}\left(\beta_{1} Q_{1}, \ldots, \beta_{N} Q_{N}\right), \quad \mathcal{R}=\operatorname{diag}\left(\beta_{1} R_{1}, \ldots, \beta_{N} R_{N}\right)
$$

Die Wichtungskoeffizienten $\beta_{i}, i=1, \ldots, N$ ermöglichen verschiedene Bewertungen der individuellen Regelgüte in einer Gesamtfunktion ${ }^{2} J=\sum_{i=1}^{N} \beta_{i} J_{i}$.

${ }^{2}$ Als Optimierungskriterium könnte z.B. auch die differenzierbare Approximation einer entwurfsparametrierten Maximumfunktion [13] verwendet werden: $J=\frac{1}{20} \ln \sum_{i=1}^{N} \exp \left(20 \cdot J_{i} / d_{i}\right)$, mit den Entwurfsvorgabewerten $d_{i}>0$. 
Die Lösung der obigen Berechnungsaufgabe ist eine Standardaufgabe des Abschnitts 2 für die expandierte Form eines Multimodellproblems. Das heißt, wir betrachten jetzt das Gesamtsystem

$$
\begin{aligned}
\lambda x(t) & =\mathcal{A} x(t)+\mathcal{B} u(t) \\
y(t) & =\mathcal{C} x(t),
\end{aligned}
$$

wobei $x(t)=\left[x_{1}^{T}(t), \ldots, x_{N}^{T}(t)\right]^{T} \in \mathbb{R}^{n N}, u(t)=\left[u_{1}^{T}(t), \ldots, u_{N}^{T}(t)\right]^{T} \in \mathbb{R}^{m N}, y(t)=$ $\left[y_{1}^{T}(t), \ldots, y_{N}^{T}(t)\right]^{T} \in \mathbb{R}^{p N}$ und $\mathcal{A}, \mathcal{B}, \mathcal{C}$ blockdiagonale Matrizen der Form

$$
\mathcal{A}=\operatorname{diag}\left(A_{1}, \ldots, A_{N}\right), \quad \mathcal{B}=\operatorname{diag}\left(B_{1}, \ldots, B_{N}\right), \quad \mathcal{C}=\operatorname{diag}\left(C_{1}, \ldots, C_{N}\right)
$$

sind. Die Rückführmatrix $F$ im Regelgesetz

$$
u_{i}(t)=F y_{i}(t), \quad i=1, \ldots, N
$$

soll alle $N$ Teilsysteme (9) simultan stabilisieren. (Es wird vorausgesetzt, daß eine solche Matrix existiert.) Für das Gesamtsystem (16) entspricht das der Bestimmung einer dezentralen, stabilisierenden Ausgangsrückkopplung mit einer blockdiagonalen Gesamtrückführmatrix $\mathcal{F}$

$$
\mathcal{F}=\operatorname{diag}(F, \ldots, F) .
$$

Die Minimierung von $J$ mit der Ausgangsrückkopplung $u(t)=\mathcal{F} y(t)$ für das Gesamtsystem (16) ist ein Standardproblem mit stark strukturierter Ausgangsrückführmatrix (19). Anhand der Ergebnisse des Abschnitts 2 kann man explizite Ausdrücke für die Auswertung der Gütefunktion und deren Gradienten herleiten. Dies ist die Aussage des folgenden Satzes.

Satz 1 (kontinuierliches System). Gegeben sei eine Rückführmatrix $F$, die mit (18) das kontinuierliche Multimodellsystem (9) stabilisiert. Dann gelten zur Auswertung der Gütefunktion $J(F)$ in (13) und deren Gradienten $\nabla J(F)$ die folgenden Formeln:

$$
\begin{aligned}
J(F) & =\sum_{i=1}^{N} \beta_{i} \operatorname{tr}\left(P_{i} G_{i}\right) \\
\nabla_{F} J(F) & =2 \sum_{i=1}^{N} \beta_{i}\left(B_{i}^{T} P_{i}+R_{i} F C_{i}\right) S_{i} C_{i}^{T} .
\end{aligned}
$$

Dabei erfüllen die Matrizen $P_{i}$ und $S_{i}$ die kontinuierlichen Liapunovgleichungen

$$
\begin{aligned}
& \bar{A}_{i}^{T} P_{i}+P_{i} \bar{A}_{i}+\bar{Q}_{i}=0 \\
& \bar{A}_{i} S_{i}+S_{i} \bar{A}_{i}^{T}+G_{i}=0
\end{aligned}
$$

$\operatorname{mit} \bar{A}_{i}=A_{i}+B_{i} F C_{i}$ und $\bar{Q}_{i}=Q_{i}+C_{i}^{T} F^{T} R_{i} F C_{i}$.

Die Lösung hat die gleiche Struktur wie im Fall eines Einzelmodells, siehe Abschnitt 2 .

Beweis. Sei $\mathcal{F}$ eine Matrix der Form

$$
\mathcal{F}=\operatorname{diag}\left(F_{1}, \ldots, F_{N}\right)
$$


wobei $F_{i}$ eine stabilisierende Ausgangsrückführmatrix für das $i$-te System ist. Seien $\mathcal{P}$ und $\mathcal{S}$ die Lösungen der kontinuierlichen Liapunovgleichungen

$$
\begin{aligned}
\overline{\mathcal{A}}^{T} \mathcal{P}+\mathcal{P} \overline{\mathcal{A}}+\overline{\mathcal{Q}} & =0 \\
\overline{\mathcal{A}} \mathcal{S}+\mathcal{S} \overline{\mathcal{A}}^{T}+\mathcal{G} & =0
\end{aligned}
$$

mit $\overline{\mathcal{A}}=\mathcal{A}+\mathcal{B} \mathcal{F} \mathcal{C}, \overline{\mathcal{Q}}=\mathcal{Q}+\mathcal{C}^{T} \mathcal{F}^{T} \mathcal{R} \mathcal{F} \mathcal{C}$ und $\mathcal{G}=\operatorname{diag}\left(G_{1}, \ldots, G_{N}\right)$. Dann kann man die Formeln [24] für eine dezentrale Rückführung anwenden

$$
\begin{aligned}
J\left(F_{1}, \ldots, F_{N}\right) & =\operatorname{tr}(\mathcal{P G}) \\
\nabla_{F_{i}} J\left(F_{1}, \ldots, F_{N}\right) & =\left[2\left(\mathcal{B}^{T} \mathcal{P}+\mathcal{R} \mathcal{F} \mathcal{C}\right) \mathcal{S C}^{T}\right]_{i i}
\end{aligned}
$$

wobei $[\cdot]_{i i}$ den $i$-ten $n \times n$ Diagonalblock einer Matrix bezeichnet. Sowohl $\overline{\mathcal{A}}$ als auch $\overline{\mathcal{Q}}$ sind blockdiagonale Matrizen, so daß jedes Blockelement $\mathcal{P}_{i j}$ außer der Blockdiagonale von $\mathcal{P}(i \neq j)$ eine Sylvestergleichung

$$
\bar{A}_{i}^{T} \mathcal{P}_{i j}+\mathcal{P}_{i j} \bar{A}_{j}=0
$$

erfüllt. Weil sowohl $\bar{A}_{i}$ als auch $\bar{A}_{j}$ stabile Matrizen sind, hat die obige Sylvestergleichung die eindeutige Lösung $\mathcal{P}_{i j}=0$. Dieselbe Folgerung gilt für die außerdiagonalen Blockelemente $\mathcal{S}_{i j}$ von $\mathcal{S}$. Wir erhalten damit die vereinfachten Ausdrücke für die Gütefunktion (13) und ihren Gradienten

$$
\begin{aligned}
J\left(F_{1}, \ldots, F_{N}\right) & =\sum_{i=1}^{N} \beta_{i} \operatorname{tr}\left(P_{i} G_{i}\right) \\
\nabla_{F_{i}} J\left(F_{1}, \ldots, F_{N}\right) & =2 \beta_{i}\left(B_{i}^{T} P_{i}+R_{i} F_{i} C_{i}\right) S_{i} C_{i}^{T},
\end{aligned}
$$

wobei $P_{i}$ und $S_{i}$ die kontinuierlichen Liapunovgleichungen (22) erfüllen mit $\bar{A}_{i}=A_{i}+$ $B_{i} F_{i} C_{i}$ und $\bar{Q}_{i}=Q_{i}+C_{i}^{T} F_{i}^{T} R_{i} F_{i} C_{i}$. Setzen wir nun alle Diagonalblöcke von $\mathcal{F}$ gleich $F_{1}=\cdots=F_{N}=F$ folgt (21) unter Anwendung folgender Beziehung

$$
\nabla_{F} J(F)=\left.\sum_{i=1}^{N} \nabla_{F_{i}} J\left(F_{1}, \ldots, F_{N}\right)\right|_{F_{1}=\cdots=F_{N}=F}=2 \sum_{i=1}^{N} \beta_{i}\left(B_{i}^{T} P_{i}+R_{i} F C_{i}\right) S_{i} C_{i}^{T}
$$

q.e.d.

Ein entsprechender Satz gilt für diskrete Systeme:

Satz 2 (diskretes System). Gegeben sei eine Rückführmatrix F, die mit (18) das diskrete Multimodellsystem (9) stabilisiert. Dann gelten zur Auswertung der Gütefunktion $J(F)$ in $(14)$ und deren Gradienten $\nabla J(F)$ die folgenden Formeln:

$$
\begin{aligned}
J(F) & =\sum_{i=1}^{N} \beta_{i} \operatorname{tr}\left(P_{i} G_{i}\right) \\
\nabla_{F} J(F) & =2 \sum_{i=1}^{N} \beta_{i}\left(B_{i}^{T} P_{i} \bar{A}_{i}+R_{i} F C_{i}\right) S_{i} C_{i}^{T} .
\end{aligned}
$$

Dabei erfüllen die Matrizen $P_{i}$ und $S_{i}$ die diskreten Liapunovgleichungen

$$
\begin{aligned}
P_{i} & =\bar{A}_{i}^{T} P_{i} \bar{A}_{i}+\overline{Q_{i}} \\
S_{i} & =\bar{A}_{i} S_{i} \bar{A}_{i}^{T}+G_{i}
\end{aligned}
$$


$\operatorname{mit} \bar{A}_{i}=A_{i}+B_{i} F C_{i}$ und $\bar{Q}_{i}=Q_{i}+C_{i}^{T} F^{T} R_{i} F C_{i}$.

Beweis. Die Beweisführung ist analog zum kontinuierlichen Fall.

Der wesentliche numerische Aufwand zur Bestimmung einer optimalen Ausgangsrückkopplung im Multimodellfall besteht in der Lösung von $N$ Paaren von Liapunovgleichungen für jede Auswertung der Funktion und ihres Gradienten. Eine Berechnung mit obigen Formeln ist offensichlich gut parallelisierbar.

Die simultane Stabilisierung mittels Ausgangsrückkopplung ist kein konvexes Minimierungsproblem. Deshalb können LMIs (engl. Linear Matrix Inequalities)-Verfahren [3] nicht direkt zur Berechnung solcher Ausgangsrückkopplungen eingesetzt werden. Man kann diese Verfahren jedoch anwenden, um die Stabilität eines polytopischen Systems zu überprüfen. Dazu bilden die folgenden "LMIs" für $i=1, \ldots, N$ für das polytopische System (11) und eine berechnete Rückführmatrix $F$ ein hinreichendes (im allgemeinen konservatives) Stabilitätskriterium:

- für ein zeitkontinuierliches System

$$
\begin{aligned}
\left(A_{i}+B_{i} F C_{i}\right) X+X\left(A_{i}+B_{i} F C_{i}\right)^{T} & <0 \\
X & >0
\end{aligned}
$$

- für ein zeitdiskretes System

$$
\begin{array}{r}
\left(A_{i}+B_{i} F C_{i}\right) X\left(A_{i}+B_{i} F C_{i}\right)^{T}-X<0 \\
X>0 .
\end{array}
$$

Haben die obigen LMIs eine Lösung $X$, ist das polytopische System (11) stabil für beliebig schnelle Änderungen im Matrizenpolytop (12). Haben diese LMIs keine gemeinsame Lösung $X$, kann gegebenfalls das Multimodellproblem durch Hinzunahme weiterer Modelle an die Robustheitsaufgabe so angepaßt werden, daß mit diesem (nur hinreichenden) Kriterium Stabilität garantiert werden kann.

\section{Periodische Multimodell-Ausgangsrückkopplung}

Diskret-periodische Ausgangsrückkopplung bietet eine Möglichkeit zur simultanen Stabilisierung konstanter Multimodellsysteme [9, 27]. Es ist deshalb auch interessant, den Lösungsansatz aus Abschnitt 3 auf diskret-periodische Ausgangsregelungen zu erweitern.

Zur Vereinfachung der Notation bei periodischen Systemen verwenden wir die ScriptNotation $\mathcal{X}$, die einer zyklischen Folge von Matrizen $X(t), t=0, \ldots, K-1$, die blockdiagonale Matrix

$$
\mathcal{X}=\operatorname{diag}(X(0), X(1), \ldots, X(K-1))
$$

zuordnet. Diese Notation ist konsistent mit den Standardmatrixoperationen Addition, Multiplikation, Invertierung. Wir notieren weiter mit $\sigma \mathcal{X}$ die $K$-zyklische Verschiebung der Folge $X(t), t=0, \ldots, K-1$

$$
\sigma \mathcal{X}=\operatorname{diag}(X(1), \ldots, X(K-1), X(0))
$$

Das Multimodell eines diskreten, linearen, periodischen Systems mit der Periode $K$ wird durch 


$$
\begin{aligned}
x_{i}(t+1) & =A_{i}(t) x_{i}(t)+B_{i}(t) u_{i}(t), \\
y_{i}(t) & =C_{i}(t) x_{i}(t)
\end{aligned},
$$

beschrieben mit $A_{i}(t)=A_{i}(t+K), B_{i}(t)=B_{i}(t+K), C_{i}(t)=C_{i}(t+K)$ für $\forall t$ und $i=1, \ldots, N$. Es gelte wieder die Annahme, daß $x_{i}(0)$ ein Zufallsvektor mit gegebener Kovarianz $G_{i}=E\left\{x_{i}(0) x_{i}^{T}(0)\right\}$ ist.

Die Skript-Notation erlaubt es, die Systemmatrizen des periodischen Multimodells in der kompakten Form

$$
\mathcal{S}_{i}=\left[\begin{array}{cc}
\mathcal{A}_{i} & \mathcal{B}_{i} \\
\mathcal{C}_{i} & 0
\end{array}\right], \quad i=1, \ldots, N
$$

zu schreiben, wobei $\mathcal{A}_{i}=\operatorname{diag}\left(A_{i}(0), A_{i}(1), \ldots, A_{i}(K-1)\right)$ und $\mathcal{B}_{i}, \mathcal{C}_{i}$ entsprechend expandierte Blockdiagonalmatrizen sind.

Die Aufgabe ist es nun, eine optimale periodische Ausgangsrückkopplung

$$
y_{i}(t)=F(t) u_{i}(t), \quad i=1, \ldots, N
$$

mit der Periode $K$, d.h. $F(t)=F(t+K) \forall t$ zu bestimmen, die simultan alle $N$ Systeme (25) Floquet-stabilisiert [21] und dabei ein Gütefunktional der Form (14) minimiert, wobei $Q_{i}(t) \geq 0$ und $R_{i}(t)>0$ jetzt symmetrische periodische Matrizen mit der Periode $K$ sind. Dabei wird vorausgesetzt, daß eine simultan stabilisierende periodische Rückführmatrix existiert.

Explizite Ausdrücke für die Gütefunktion und den Gradienten wurden in [26] für den Fall eines diskreten periodischen Einzelsystems hergeleitet. Damit können die Ergebnisse des Abschnitts 3 im folgenden Satz auf den Fall von Multimodellsystemen mit diskretperiodischer Ausgangsrückkopplung erweitert werden.

Satz 3 (diskretes periodisches System). Sei $F(t)$ eine diskret-periodische Rückführmatrix in (26), die alle Trägersysteme in (25) simultan Floquet-stabilisiert. Das Gütefunktional $J(\mathcal{F})=\sum_{i=1}^{N} \beta_{i} J_{i}(\mathcal{F})$ für das Gesamtsystem und dessen Gradienten $\nabla J(\mathcal{F})$ können mit den Formeln

$$
\begin{aligned}
J(\mathcal{F}) & =\sum_{i=1}^{N} \beta_{i} \operatorname{tr}\left(\sigma \mathcal{P}_{i} \mathcal{G}_{i}\right) \\
\nabla_{\mathcal{F}} J(\mathcal{F}) & =2 \sum_{i=1}^{N} \beta_{i}\left(\mathcal{B}_{i}^{T} \sigma \mathcal{P}_{i} \overline{\mathcal{A}}_{i}+\mathcal{R}_{i} \mathcal{F} \mathcal{C}_{i}\right) \mathcal{S}_{i} \mathcal{C}_{i}^{T}
\end{aligned}
$$

ausgewertet werden, wobei $\mathcal{P}_{i}$ und $\mathcal{S}_{i}$ die diskret-periodischen Rückwärts- und bzw. VorwärtsLiapunovgleichungen

$$
\begin{aligned}
& \mathcal{P}_{i}=\overline{\mathcal{A}}_{i}^{T} \sigma \mathcal{P}_{i} \overline{\mathcal{A}}_{i}+\overline{\mathcal{Q}}_{i}, \\
& \sigma \mathcal{S}_{i}=\overline{\mathcal{A}}_{i} \mathcal{S}_{i} \overline{\mathcal{A}}_{i}^{T}+\mathcal{G}_{i},
\end{aligned}
$$

erfüllen mit $\overline{\mathcal{A}}_{i}=\mathcal{A}_{i}+\mathcal{B}_{i} \mathcal{F C}_{i}, \overline{\mathcal{Q}}_{i}=\mathcal{Q}_{i}+\mathcal{C}_{i}^{T} \mathcal{F}^{T} \mathcal{R}_{i} \mathcal{F} \mathcal{C}_{i}$, und $\mathcal{G}_{i}=\operatorname{diag}\left(0,0, \ldots, G_{i}\right)$.

Dies beinhaltet auch den Fall, daß für ein nichtperiodisches zeitinvariantes System mit $\mathcal{A}_{i}=\operatorname{diag}\left(A_{i}, \ldots, A_{i}\right), \mathcal{B}_{i}=\operatorname{diag}\left(B_{i}, \ldots, B_{i}\right), \mathcal{C}_{i}=\operatorname{diag}\left(C_{i}, \ldots, C_{i}\right)$ mit konstanten Matrizen $A_{i}, B_{i}, C_{i}, i=1, \ldots, N$, eine diskret-periodische Ausgangsrückkopplung (26) bestimmt werden kann. Dies ist deshalb interessant, weil eine diskret-periodische 
Ausgangsrückkopplung ein höheres "Stabilisierungspotential" hat als eine konstante Ausgangsrückkopplung.

Der wesentliche numerische Aufwand zur Bestimmung einer optimalen periodischen Ausgangsrückkopplung im Multimodellfall besteht jetzt in der Lösung von $N$ Paaren periodischer Liapunovgleichungen für jede Auswertung der Funktion und des Gradienten. Effiziente und numerisch zuverlässige Verfahren zur Lösung diskret-periodischer Liapunovgleichungen der Form (27) und (28) wurden vor kurzem entwickelt und implementiert [25]. Damit ist auch die Lösung "großer" Multimodellprobleme möglich, die durch eine große Periodenzahl $K$ entstehen.

\section{Anwendung: Robuste Stabilisierung der Hubschrauberbodenresonanz}

Bei Eurocopter wird derzeit untersucht, ob die passive Hubschrauber-Rotorblattdämpfung durch eine aktive Stabilisierung mit Ausgangsrückkopplung ersetzt werden könnte. Dafür war zu untersuchen, ob die Aufgabe durch eine robuste Regelung lösbar ist, d.h. ob durch eine konstante Ausgangsrückkopplung die Bodenresonanzeffekte im gesamten Arbeitsbereich von Rotordrehzahl und Schub genügend gedämpft werden können.

Dazu wurde das Modell der nichtlinearen Aeromechanik des Hubschraubers linearisiert und zwar [22] für 31 unterschiedliche Drehzahlen im Bereich von $80 \%$ bis $110 \%$ der Nenndrehzahl sowie für 10 Schubwerte im Bereich von 0 bis $100 \%$ des Maximalschubs bei $98 \%$ der Nenndrehzahl (Bodenresonanz). Jedes linearisierte HubschrauberAeromechanikmodell hat 24 Zustandsgrößen, 2 Stelleingänge und 4 Ausgangsgrößen. Dabei enthält jedes Modell 2 Verzögerungsglieder 1. Ordnung als Stellgliedmodelle und 2 Bandpaßfilter 2. Ordnung. Der Zweck der Filter ist es, die Reglerwirksamkeit auf die Umgebung der Resonanzfrequenz, das ist ungefähr $10 \mathrm{~Hz}$, zu begrenzen. Bild 1 zeigt, daß das ungeregelte System im Bereich des Resonanzpunkts (98 \% der Rotordrehzahl) für praktisch alle Schubwerte aeromechanisch instabil ist, d.h. Dämpfungswerte kleiner null aufweist.

Diese Aufgabe einer robusten Stabilisierung wurde nach Abschnitt 3 als Multimodellproblem mit quadratisch-optimaler Ausgangsrückkopplung gelöst. Dazu wurden aus der Menge der 41 verfügbaren Modelle zunächst nur eine Untermenge von 7 Modellen verwendet. Dies waren Modelle für die Rotordrehzahlen $80 \%, 90 \%, 98$ \%, $104 \%$ und $110 \%$ Nenndrehzahl sowie Modelle für $30 \%$ und $50 \%$ Maximalschub. Die erreichte Dämpfung des geregelten Systems ist in Bild 1 im Vergleich zum ungeregelten System gezeigt; gefordert war eine Mindestdämpfung 0,015 über alle Drehzahlen. Die erforderliche Rechenzeit zur simultanen Stabilisierung der ursprünglich instabilen Modelle und zur Minimierung der quadratischen Gütefunktionale betrug auf einer Workstation HP 9000/715 (80MHz) weniger als 1 Minute.

Die Anzahl der Modelle, die simultan berücksichtigt werden, kann variiert werden. Durch Hinzunahme weiterer Modelle können im Verlauf einer Untersuchung kritische Bereiche gezielter analysiert werden. Andererseits kann die Regelgüte in den einzelnen Linearisierungspunkten durch unterschiedliche Wichtungen in den Gütefunktionen beein- 

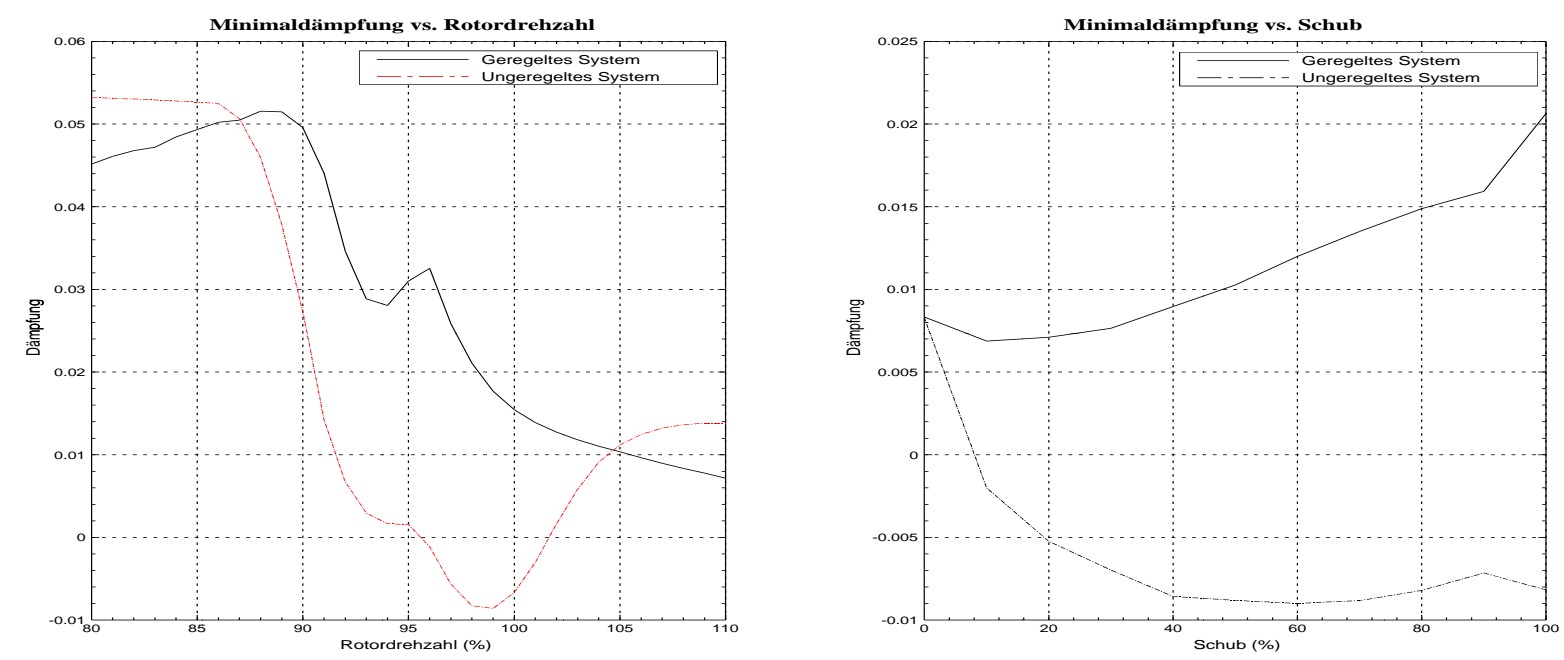

Abbildung 1: Minimaldämpfung vs. Rotordrehzahl und Schub (gleichmäßige Gewichtung im Gütefunktional)

flußt werden. Auf diese Weise wurde das noch nicht befriedigende Ergebnis in Bild 1 für Rotordrehzahlen oberhalb der Nominaldrehzahl verbessert: Bild 2 zeigt Ergebnisse, die durch eine unterschiedliche Wichtung erreicht worden sind, wobei die Güte insbesondere für höhere Schubwerte verbessert werden sollte. Dabei wurde gleichzeitig auch die Anzahl der simultan behandelten Modelle auf 15 erhöht. Es zeigt sich, daß die Dämpfungswerte über alle Rotordrehzahlen und bei höherem Schub verbessert wurden, allerdings im Kompromiß mit schlechteren Dämpfungswerten für niedrigen Schub.

Die Methode ist als interaktiver Berechnungsmodul in der regelungsdynamischen Entwurfsumgebung ANDECS [6] verfügbar.

\section{Zusammenfassung}

Der Aufsatz behandelt eine Vorgehensweise zur numerischen Optimierung simultan stabilisierender Ausgangsrückkopplungen für lineare, zeitkontinuierliche oder zeitdiskrete Multimodellsysteme, wie dies für eine arbeitspunktrobuste Regelung notwendig ist. Die dazu angewandte Methode ist die lineare quadratisch-optimale Ausgangsrückkopplung. Ein Vorteil dieser Methode ist, daß unterschiedliche Modelle, die durch Linearisierung eines nichtlinearen Systemmodells in unterschiedlichen Arbeitspunkten gegeben sind, durch unterschiedliche Wichtungen im Gütefunktional unterschiedlich bewertet werden können. Damit kann neben einer Stabilisierung auch die Regelgüte gezielt beeinflußt werden. Hinzu kommt, daß die für eine iterative Minimierung der Gütefunktion notwendigen Auswertungen des Funktionswerts und des Gradienten numerisch effektiv durch Lösen von Liapunovgleichungen erfolgen kann. Der numerische Aufwand besteht im wesentlichen in der Lösung von N Paaren von Liapunovgleichungen pro Minimierungsschritt, wenn N Modelle simultan behandelt werden. Die Vorgehensweise kann sowohl für zeitinvariante (dynamische) Ausgangsrückkopplung als auch für diskret-periodische Ausgangsrückkopplung angewendet werden. Zur Auswertung der Liapunovgleichungen steht in beiden Fällen 

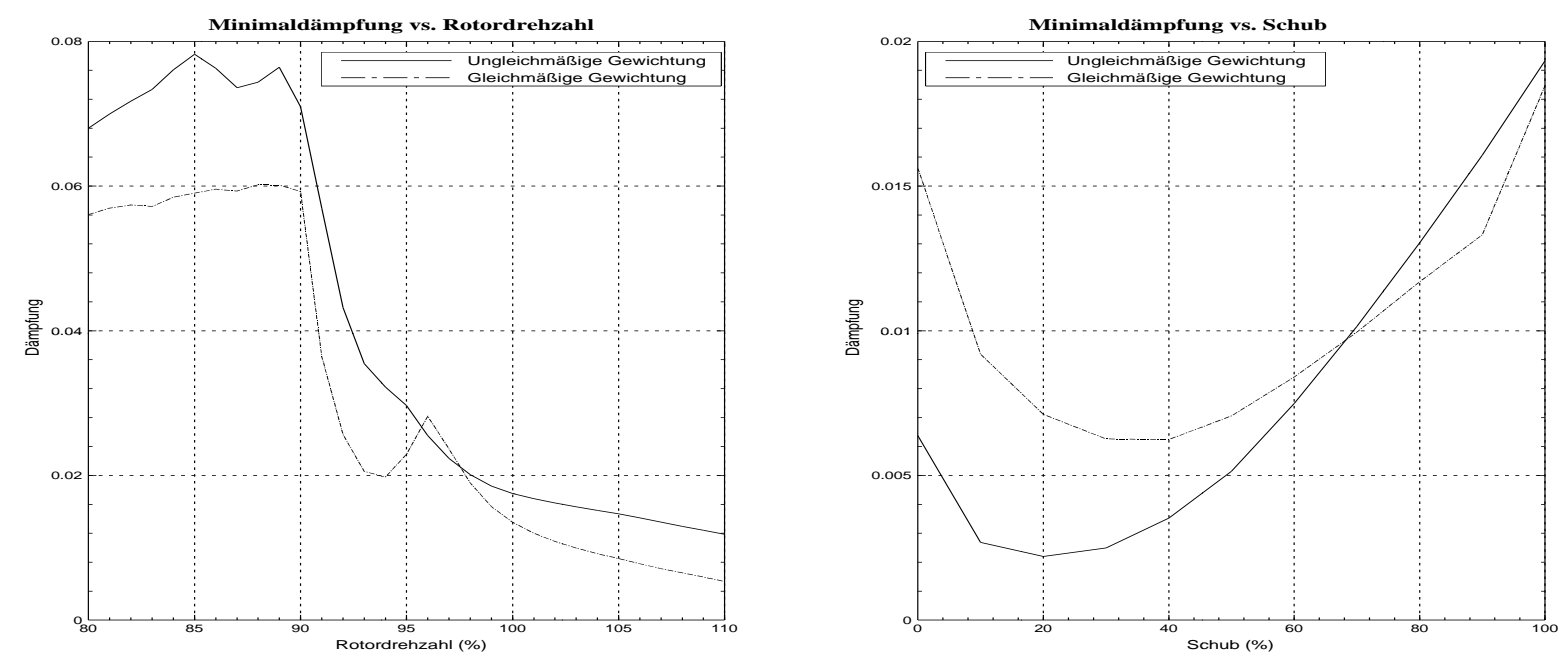

Abbildung 2: Minimaldämpfung vs. Rotordrehzahl und Schub (ungleichmäßige Gewichtung im Gütefunktional)

effiziente Software zur Verfügung. Das gleiche gilt für die hier eingesetzten Minimierungsverfahren mit Schrittweitensteuerung.

Die Existenz einer simultan stabilisierenden Ausgangsrückkopplung kann, wie bekannt, a priori nicht immer vorausgesetzt werden. Dies gilt bereits für die Stabilisierbarkeit von Einzelmodellen. Wie aber anhand der praktischen Anwendung des Verfahrens zur aktiven Bodenresonanzdämpfung für Hubschrauberrotoren demonstriert wurde, kann dieses numerische Verfahren durchaus erfolgreich auch für "große" Probleme eingesetzt werden. Dabei wurden 17 Modelle von jeweils 24. Ordnung simultan stabilisiert und optimiert.

Die in diesem Beitrag durchgeführte Erweiterung auf diskret periodische Ausgangsrückführungen ist insbesondere auch für die simultane Stabilisierung von Multimodellsystemen mit konstanten (nichtperiodischen) Streckenparametern interessant, weil periodische Ausgangsrückkopplungen bereits bei Einzelsystemen ein erhöhtes Stabilisierungspotential aufweisen.

\section{Schrifttum}

[1] Ackermann, J.: Robuste Regelung. Springer Verlag Berlin, 1993.

[2] Bartels, R. H., und Stewart, G. W.: Algorithm 432: Solution of the matrix equation $\mathrm{AX}+\mathrm{XB}=\mathrm{C}$. Comm. ACM, 15 (1972) S. 820-826.

[3] Boyd, S., El Ghaoui, L., Feron, E., und Balakrishnan, V.: Linear Matrix Inequalities in System and Control Theory. SIAM, 1994.

[4] Grübel, G., und Kreisselmeier, G.: Effective parameter sensitivity reduction through minimization of a sensitivity measure. In Proc. JACC, S. 79-86, 1971.

[5] Grübel, G., Varga, A., van den Boom, A., und Geurts, A. J.: Towards a coordinated development of numerical CACSD software: the RASP/SLICOT compatibility 
concept. In Prepr. of IEEE/IFAC Symp. CACSD’94, Tucson, Arizona,S. 499-504, 1994.

[6] Grübel, G., Finsterwalder, R., Gramlich, G. Joos, H.-D. und Lewald, A., ANDECS: A Computation Environment for Control Applications of Optimization. R. Bulirsch, D. Kraft, eds. Birkhäuser, Basel, Boston, Berlin, Control Application of Optimization, International Series of Numerical Mathematics (ISNM), Vol. 115, S. 237-254, 1994.

[7] Höfler, A. B.: Gradientenkettenoperatoren und ihre Anwendung bei der Reglerparameteroptimierung. Dissertation. Schriftenreihe, Lehrstuhl für Meß- und Regelungstechnik (Prof. K.H. Fasol), Ruhr-Universität Bochum, Heft 14, 1980.

[8] Jameson, A.: Optimization of linear systems of constrained configuration. Int. J. Control, 11 (1970) S. 409-421.

[9] Kabamba, P.: Control of linear systems using generalized sampled-data hold functions. IEEE Trans. Autom. Control, 32 (1987) S. 772-783.

[10] Kalman, R. E.: Contributions to the theory of optimal control. Bol. Soc. Mat. Mexicana, (1960) S. 102-119.

[11] Kessler, Ch., und Reichert, G.: Active control of ground and air resonance including transition from ground to air. In Proc. 20th European Rotorcraft Forum, Amsterdam, S. 64.1-64.17, 1994.

[12] Kitagawa, G.: An algorithm for solving the matrix equation $X=F X F^{\prime}+S$. Int. J. Control, 25 (1977) S. 745-753.

[13] Kreisselmeier, G. und Steinhauser, R.: Systematische Auslegung von Reglern durch Optimierung eines vektoriellen Gütekriteriums. Z. Regelungstechnik 1979, H. 3, S. $76-79$.

[14] Kosut, R.L.: Suboptimal control of linear time-invariant systems subject to control structure constraints. IEEE Trans. Autom. Control, 15 (1970) S. 557-563.

[15] Kuhn, U.: Ein neuer Weg zur Bestimmung einer optimalen Ausgangsrückführung für die Regelung linearer Systeme. Automatisierungstechnik, at 33 (1985) S. 89-95.

[16] Levin, W.S. und Athans, M.: On the determination of the optimal constant output feedback gains for linear multivariable systems. IEEE Trans. Autom. Control, 15 (1970) S. 44-48.

[17] Liu, D. C., und Nocedal, J.: On the limited memory BFGS method for large scale optimization. Mathematical Programming, 45 (1989) S. 503-528.

[18] Mäkilä, P. M., und Toivonen, H. T.: Computational methods for parametric LQ problems - a survey. IEEE Trans. Autom. Control, 32 (1987) S. 658-671.

[19] Moré, J. J.: User's Guide for MINPACK-1. Applied Mathematics Division Report ANL-80-74, Argonne National Laboratory, Argonne,IL, 1980. 
[20] Moré, J. J., und Thuente, D. J.: On line search algorithms with guaranteed sufficient decrease. Mathematics and Computer Science Division Preprint MCS-P330-1092, Argonne National Laboratory, Argonne, IL, 1990.

[21] Müller, Peter C.: Stabilität und Matrizen. Springer Verlag Berlin 1977.

[22] Teves, D.: (private Mitteilung). 1996.

[23] Teves, D., Niesl, G., Blaas, A., und Jacklin, S.: The role of rotor active control in future rotorcraft. In Proc. 21st European Rotorcraft Forum, St. Petersburg, volume III, S. 10.1-10.17, 1995.

[24] Toivonen, H. T., und Mäkilä, P. M.: A descent Anderson-Moore algorithm for optimal decentralized control. Automatica, 21 (1985) S. 743-744.

[25] Varga, A.: Periodic Lyapunov equations: some applications and new algorithms. Technical Report TR R197-96, DLR-Oberpfaffenhofen, Institut für Robotik and Systemdynamik, 1996. (eingereicht für Int. J. Control).

[26] Varga, A., und Pieters, S.: A computational approach for optimal periodic output feedback control. In Proc. CACSD’96 Symposium, Dearborn, MI, 1996, S. 176-181.

[27] Werner, H., und Furuta, K.: Simultaneous stabilization by piecewise constant periodic output feedback. Control Theory and Advanced Technology, 10 No. 4, Part 4. (1987) S. 1763-1775. 